Использование метода главных компонент для совершенствования профилактической работы с факторами риска сердечно-сосудистых заболеваний

\author{
И.В. Ковригина ${ }^{1}$, Е.В. Болотова ${ }^{2}$, А.В. Концевая ${ }^{3}$ \\ 1 ГБуз «Научно-исследовательский институт - Краевая клиническая больница № 1 имени профессора С. В. Очаповского» \\ Министерства здравоохранения Краснодарского края; Россия, г. Краснодар \\ 2 ФГБоу ВО «Кубанский государственный медицинский университет» Министерства здравоохранения Российской Федерации; \\ Россия, г. Краснодар \\ ${ }^{3}$ ФГБУ «Национальный медицинский исследовательский центр профилактической медицины» Министерства здравоохранения \\ Российской Федерации; Россия, г. Москва
}

\title{
PЕЗЮME
}

\begin{abstract}
Цель исследования: повышение эффективности профилактики сердечно-сосудистых заболеваний (ССЗ) у пациентов IIIa группы здоровья в территориальной поликлинике г. Краснодара на основе факторного анализа (метод главных компонент) результатов 1-го этапа диспансеризации в 2015 и 2018 г.

Материалы и методы. В исследование включены лица, прошедшие 1-й этап диспансеризации: в 2015 г. - 2461, а в 2018 г. 2772 пациента. У больных с IIIа группой здоровья изучены модифицируемые факторы риска (ФР) ССЗ с использованием метода главных компонент.

Результаты. В 2015 г. IIIа группа здоровья определена у 29,9\% пациентов; доля женщин - 68,5\% (медиана возраста - 62 года), доля мужчин - 31,5\% (медиана возраста - 60 лет). В 2018 г. IIIа группа здоровья зафиксирована у 41,5\% пациентов; доля женщин - 64,3\% (медиана возраста - 63 года), доля мужчин - 35,7\% (медиана возраста - 62 года). По данным факторного анализа, у мужчин имеются следующие сочетания модифицируемых ФР ССЗ: фактор 1 - нерационально питание (НП) и низкая физическая активность (НФА); фактор 2 - гиперхолестеринемия (ГХС), курение табака и риск пагубного потребления алкоголя (РППА); фактор 3 артериальная гипертензия (АГ) и НП; фактор 4 - АГ. У женщин найдены несколько другие сочетания ФР ССЗ: фактор 1 - НП и НФА; фактор 2 - АГ и ГХС; фактор 3 - индекс массы тела > 25 кг/м² и гипергликемия; фактор 4 - РППА.

Заключение. Выявленные с помощью метода главных компонент гендерные различия в сочетании модифицируемых ФР ССЗ у пациентов c IIIа группой здоровья позволяют проводить мероприятия по коррекции ФР с учетом сочетаний, оказывающих наиболее значимое влияние на наступление неблагоприятных сердечно-сосудистых событий.

Ключевые слова: диспансеризация, фактор риска, сердечно-сосудистые заболевания, профилактика, поликлиника, факторный анализ.
\end{abstract}

Вклад авторов: Болотова Е.В. - разработка концепции и дизайна исследования, написание текста рукописи, утверждение рукописи для публикации; Ковригина И.В. - сбор и обработка материала, статистическая обработка, написание текста рукописи; Концевая А.В. редактирование статьи.

Конфликт интересов: авторы заявляют об отсутствии возможных конфликтов интересов.

Для цитирования: Ковригина И.В., Болотова Е.В., Концевая А.В. Использование метода главных компонент для совершенствования профилактической работы с факторами риска сердечно-сосудистых заболеваний. Доктор.Ру. 2021; 20(4): 7-14. DOI: 10.31550/1727-2378-2021-20-4-7-14

\section{Use of the Dominant Component Analysis for Improvement of Preventive Measures in Patients with Cardiovascular Risks}

\section{I.V. Kovrigina ${ }^{1}$, E.V. Bolotova ${ }^{2}$, A.V. Kontsevaya ${ }^{3}$}

${ }^{1}$ Scientific and Research Institute - Regional Clinical Hospital No.1 named after Professor S.V. Ochapovsky of Krasnodar Territorial Ministry of Health; 167 1-go Maya Str., Krasnodar, Russian Federation 350086

${ }^{2}$ Kuban State Medical University of the Ministry of Health of the Russian Federation; 4 Mitrofan Sedin Str., Krasnodar, Russian Federation 350063

${ }^{3}$ National Medical Research Centre of Preventive Medicine of the Ministry of Health of the Russian Federation; 10 Petroverigsky per., Moscow, Russian Federation 101990

Ковригина Ирина Валерьевна (автор для переписки) - заведующая амбулаторно-поликлиническим отделением для прикрепленного населения г. Краснодара ГБУЗ «НИИ - ККБ № 1 им. проф. С.В. Очаповского» Минздрава Краснодарского края. 350086, Россия, г. Краснодар, ул. 1-го Мая, д. 167. https://orcid.org/0000-0001-9966-8905. E-mail: kovriginairina2010@mail.ru

Болотова Елена Валентиновна - д. м. н., профессор кафедры терапии № 1 факультета повышения квалификации и профессиональной переподготовки специалистов ФГБоу Во КубГму Минздрава России. 350063, Россия, г. Краснодар, ул. им. Митрофана Седина, д. 4. eLIBRARY.RU SPIN: 4322-9985. https://orcid.org/0000-0001-6257-354X.E-mail: bolotowa_e@mail.ru

Концевая Анна Васильевна - Ә. м. н., заместитель директора по научной и аналитической работе ФГБУ «Нмиц профилактической медицины» Минздрава России. 101990, Россия, г. Москва, Петроверигский пер., д. 10. eLIBRARY.RU SPIN: 6787-2500. https://orcid.org/00000003-2062-1536. E-mail: koncanna@yandex.ru 


\section{ABSTRACT}

Study Objective: To improve efficiency of cardiovascular disease (CVD) prevention in patients with health category IIIa in Krasnodar Territorial Outpatient Clinic using a factorial analysis (dominant component analysis) of stage 1 health assessment results for 2015 and 2018. Materials and Methods. The study includes subjects who underwent stage 1 health assessment: $2015-2,461$ people, $2018-2,772$ people. Patients with health category IIIa were assessed for modifiable risk factors (RF) of CVDs using the dominant component analysis.

Study Results. In 2015, health category IIIa was assigned to $29.9 \%$ of patients; female patients accounted for $68.5 \%$ (median age: 62 years), males accounted for $31.5 \%$ (median age: 60 years). In 2018, health category IIIa was recorded for $41.5 \%$ of patients; female patients accounted for $64.3 \%$ (median age: 63 years), males accounted for $35.7 \%$ (median age: 62 years). Factorial analysis demonstrated that male patients have the following composition of modifiable CVD RFs: factor 1 - improper diet (ID) and physical inactivity (PIA); factor 2 hypercholesterolemia (HCS), tobacco smoking and alcohol abuse (TSAA); factor 3 - arterial hypertension (AH) and ID; factor $4-\mathrm{AH}$. Women demonstrated different compositions of CVD RFs: factor 1 - ID and PIA; factor $2-\mathrm{AH}$ and HCS; factor $3-\mathrm{BMI}>25 \mathrm{~kg} / \mathrm{m}^{2}$ and hyperglycemia; factor 4 - TSAA.

Conclusion. Gender differences together with modifiable CVD RFs in patients with risk category IIIa revealed with the help of the dominant component analysis allow correcting RFs taking into account combinations, most contributing to unfavourable cardiovascular events.

Keywords: health assessment, risk factor, circulatory diseases, prevention, outpatient clinic, factorial analysis.

Contributions: Bolotova, E.V. — study concept and design; text of the article, approval of the manuscript for publication; Kovrigina, I.V. — data collection and processing; statistical processing; text of the article; Kontsevaya, A.V. - article editing.

Conflict of interest: The authors declare that they do not have any conflict of interests.

For citation: Kovrigina I.V., Bolotova E.V., Kontsevaya A.V. Use of the Dominant Component Analysis for Improvement of Preventive Measures in Patients with Cardiovascular Risks. Doctor.Ru. 2021; 20(4): 7-14. (in Russian). DOI: 10.31550/1727-2378-2021-20-4-7-14

\section{ВВЕДЕНИЕ}

В глобальном плане действий по профилактике хронических неинфекционных заболеваний (ХНИЗ) и борьбе с ними прописана амбициозная цель - снижение преждевременной смертности от ХНИЗ на 25\% к 2025 году. Для достижения этой цели подчеркивается особая важность уменьшения распространенности среди отдельных лиц и групп населения общих модифицируемых факторов риска (ФР) неинфекционных заболеваний, а также создания необходимого потенциала для ведения здорового образа жизни [1].

В России, несмотря на достижение определенных положительных результатов [2], смертность от болезней системы кровообращения остается одной из самых высоких в мире, чем обусловлен суммарный экономический ущерб около 2,7 трлн руб. в год (3,2\% от ВВП) [3]. На фоне столь тяжелого бремени ССЗ становится очевидной необходимость совершенствования профилактики данной группы заболеваний. Традиционно в нашей стране упор делается на двухэтапную диспансеризацию, приоритетами которой являются раннее обнаружение и своевременная коррекция модифицируемых ФР и отдельных нозологических единиц сердечно-сосудистых заболеваний (СС3) [4].

Раннее выявление и качественная коррекция таких модифицируемых ФР ССЗ, как повышенное АД, гиперхолестеринемия (ГХС), гипергликемия, курение табака, нерациональное питание (НП), избыточная масса тела (ИзМТ) и ожирение, низкая физическая активность (НФА), риск пагубного потребления алкоголя (РППА), препятствуют прогрессированию СС3, развитию их осложнений и предупреждают обусловленную ими смертность [5]. Однако имеет место значительная вариабельность показателей заболеваемости и смертности от болезней системы кровообращения по регионам РФ [2, $4,6]$. Существующие на прикрепленной к поликлинике территории местные особенности демографических процессов, состояние окружающей среды, экономики и другие факторы могут оказывать влияние на эффективность проводимых профилактических мероприятий. Особую остроту проблеме придает тот факт, что в ряде случаев сложно оценить эффективность профилактики неблагоприятных сердечно-сосу- дистых событий у пациентов IIIа группы здоровья (традиционно упор делается на II группу здоровья), так как подсчет суммарного сердечно-сосудистого риска при помощи Европейской шкалы SCORE зачастую показывает очень высокий абсолютный сердечно-сосудистый риск [7].

Таким образом, чрезвычайно актуальной задачей становится поиск новых подходов к оптимизации профилактики ССЗ с учетом роли модифицируемых ФР и их сочетаний в ходе 1-го этапа диспансеризации у пациентов IIIa группы здоровья в отдельной поликлинике.

Цель исследования: повышение эффективности профилактики ССЗ у пациентов IIIа группы здоровья в территориальной поликлинике г. Краснодара на основе факторного анализа результатов 1-го этапа диспансеризации в 2015 и 2018 году.

\section{МАТЕРИАЛЫ И МЕТОДЫ}

В исследовании использованы данные, полученные в ходе диспансеризации взрослого населения, прикрепленного по территориально-участковому принципу для оказания первичной медико-санитарной помощи к амбулаторно-поликлиническому отделению ГБУЗ «НИИ - ККБ № 1 им. проф. С.В. Очаповского» г. Краснодара. Исследование выполнено в соответствии со стандартами надлежащей клинической практики (Good Clinical Practice $)^{1}$. При включении в исследование у всех пациентов получено письменное информированное согласие. Протокол № 07-05/17 от 14.12.2017 г. одобрен на заседании независимого этического комитета ФГБУ «НМИЦ профилактической медицины» Министерства здравоохранения России.

В качестве целевой группы отобраны пациенты IIIa группы здоровья, прошедшие 1-й этап диспансеризации в 2015 и в 2018 году. С учетом гендерных и возрастных особенностей в целевой группе изучена частота следующих модифицируемых ФР ССЗ: АГ, ГХС, гипергликемии, курения табака, НП (избыточного потребления пищи, жиров, углеводов, потребления поваренной соли более 5 г в сутки (досаливания приготовленной пищи, частого употребления соленостей, консервов, колбасных изделий), недостаточного употребления фруктов и овощей - менее 400 г или менее 4-6 порций в сутки), ИзМТ и ожирения (ИМТ > 25 кг/м²), НФА,

1 ГОСТ P 52379-2005 «Надлежащая клиническая практика». Национальный стандарт Российской Федерации. M.; 2006. URL: https://docs.cntd.ru/ document/1200041147 (дата обращения - 15.03.2021). 
РППА (определяется по результатам анкетирования при прохождении диспансеризации).

Модифицируемые ФР ССЗ в ходе диспансеризации оценивали с учетом Приложения 2 Приказа Минздрава РФ от 03.02.2015 г. № 36ан «0б утверждении порядка проведения диспансеризации определенных групп взрослого населения» и Приказа Минздрава РФ от 26.10.2017 г. № 869н «0б утверждении порядка проведения диспансеризации определенных групп взрослого населения». Анализ частоты модифицируемых ФР ССЗ проведен в трех возрастных группах (1-я группа - 18-38 лет, 2-я группа - 39-60 лет, 3-я группа - 61 год и более) в соответствии с Приказом Минздрава РФ от 06.03.2015 г. № 87н «0б унифицированной форме медицинской документации и форме статистической отчетности, используемых при проведении диспансеризации определенных групп взрослого населения и профилактических медицинских осмотров, порядках по их заполнению».

Статистический анализ данных осуществлен с помощью программы Statistica 12 (StatSoft Inc., США). В связи c неоднородностью количественного состава пациентов, прошедших диспансеризацию, для описания их возраста использовали Ме (Q1-Q3), где Ме - медиана, Q1 - нижний, Q3 - верхний квартиль. Номинальные данные описывались с указанием абсолютных значений и процентных долей.

С целью проверки нулевой гипотезы об отсутствии различий между частотами распространенности модифицируемых ФР использовали критерий $\chi^{2}$ Пирсона, при $\mathrm{p} \leq 0,05$ принимали альтернативную гипотезу о наличии различий [8].

Для характеристики модифицируемых ФР ССЗ, оказывающих наиболее значимое влияние на наступление неблагоприятных сердечно-сосудистых событий, применяли разновидность факторного анализа - метод главных ком- понент [9]. Количество факторов определяли с помощью критерия Кайзера, учитывались факторы с собственным значением не менее 1 [9].

В связи с относительно низким количеством наблюдений в 1-й и 2-й возрастных группах IIIа группы здоровья, согласно «правилу $100 »$, метод главных компонент использовали только с учетом пола пациентов [10].

Для построения интерпретируемой матрицы факторных нагрузок проводилось вращение факторов методом Varimax raw. В качестве значимых для интерпретации принимались факторные нагрузки более 0,5, а высоких - 0,7 и выше [9].

\section{РЕЗУЛЬТАТЫ}

В 2015 г. 1-й этап диспансеризации прошел 2461 человек (38 (29-56) лет), среди них 66\% женщин (38 (29-57) лет), 34\% мужчин (36 (27-54) лет). По результатам 1-го этапа диспансеризации в 2015 г. IIIа группа здоровья определена у 737 (29,9\%) больных (62 (53-72) года), среди них 68,5\% женщин (62 (54-74) года), 31,5\% мужчин (60 (53-67) лет) (табл. 1,2).

В 2018 году 1-й этап диспансеризации прошли 2772 человека (47 (35-62) лет): 65,5\% женщин (48 (35-62) лет), 34,5\% мужчин (45 (33-61) лет). IIIа группа здоровья зафиксирована у 1150 (41,5\%) пациентов (63 (56-71) года): 64,3\% женщин (63 (56-71) года), 35,7\% мужчин (62 (53-69) года).

0бщее количество больных, повторно прошедших 1-й этап диспансеризации в 2018 г, составило 1170 (47,5\%; 45 (3362) лет): 69,2\% женщин (47 (35-62) лет), 30,8\% мужчин (44 (33-62) года).

Из 737 пациентов, получивших по результатам 1-го этапа диспансеризации в 2015 г. IIIa группу здоровья, в 2018 г. зарегистрирован только 371 человек (50,3\%; 65 (59-73) лет): $68,2 \%$ женщин (66 (59-77) лет), 31,8\% (64 (57-71) года).

Таблица 1 / Table 1 ?

Гендерные и возрастные особенности пациентов по Аанным 1-го этапа диспансеризации в 2015 и 2018 годах

Gender and age-related differences between patients based on stage 1 health assessment results for 2015 and 2018

\begin{tabular}{|l|l|l|l|l|l|l|}
\hline Bозраст, годы / Age, years & \multicolumn{3}{|c|}{2015 (2461) } & \multicolumn{2}{c|}{2018 (2772) } & \multicolumn{2}{c|}{$\begin{array}{c}\text { из } 2015 \text { в } 2018 \text { / from 2015 } \\
\text { to 2018 (1170) }\end{array}$} \\
\cline { 2 - 7 } & $\begin{array}{c}\text { мужчины / } \\
\text { males }\end{array}$ & $\begin{array}{c}\text { женщины / } \\
\text { females }\end{array}$ & $\begin{array}{c}\text { мужчины / } \\
\text { males }\end{array}$ & $\begin{array}{c}\text { женщины / } \\
\text { females }\end{array}$ & $\begin{array}{c}\text { мужчины / } \\
\text { males }\end{array}$ & $\begin{array}{c}\text { женщины / } \\
\text { females }\end{array}$ \\
\hline Bcero / Total & 834 & 1627 & 956 & 1816 & 360 & 810 \\
\hline $18-38$ & 468 & 825 & 351 & 595 & 138 & 285 \\
\hline $39-60$ & 234 & 490 & 364 & 728 & 125 & 301 \\
\hline 61 и старше / 61+ years old & 132 & 312 & 241 & 493 & 97 & 224 \\
\hline
\end{tabular}

Таблица 2 / Table 2

Гендерные и возрастные особенности

пациентов IIIа группы здоровья в 2015 и 2018 годах

Gender and age-related differences between patients with health category IIIa in 2015 and 2018

\begin{tabular}{|l|l|l|l|l|l|l|}
\hline Bозраст, годы / Age, years & \multicolumn{2}{|c|}{2015 (737) } & \multicolumn{2}{c|}{2018 (1150) } & \multicolumn{2}{c|}{$\begin{array}{c}\text { из 2015 в 2018 / from 2015 } \\
\text { to 2018 (371) }\end{array}$} \\
\cline { 2 - 7 } & $\begin{array}{c}\text { мужчины / } \\
\text { males }\end{array}$ & $\begin{array}{c}\text { женщины / } \\
\text { females }\end{array}$ & $\begin{array}{c}\text { мужчины / } \\
\text { males }\end{array}$ & $\begin{array}{c}\text { женщины / } \\
\text { females }\end{array}$ & $\begin{array}{c}\text { мужчины / } \\
\text { males }\end{array}$ & $\begin{array}{c}\text { женщины / } \\
\text { females }\end{array}$ \\
\hline Bcero / Total & 232 & 505 & 411 & 739 & 118 & 253 \\
\hline $18-38$ & 10 & 17 & 23 & 25 & 2 & 1 \\
\hline $39-60$ & 109 & 216 & 166 & 278 & 41 & 78 \\
\hline 61 и старше / 61+ years old & 113 & 272 & 222 & 436 & 75 & 174 \\
\hline
\end{tabular}


На фоне роста на 12,6\% количества прошедших диспансеризацию в 2018 г. наблюдается повышение доли лиц с IIIa группой здоровья с 29,9\% в 2015 г. до 41,5\% в 2018 г., соотношение мужчин и женщин сохранялось близким к $1: 2$. За трехлетний период отмечался рост медианы возраста пациентов, прошедших 1-й этап диспансеризации, на 9 лет (с 38 до 47 лет): у женщин - на 10 лет (с 38 до 48 лет), у мужчин - на 9 лет (с 36 до 45 лет). Схожая тенденция сохранялась у пациентов из IIIа группы здоровья - медиана возраста выросла на 1 год (с 62 до 63 лет): у женщин - на 1 год (с 62 до 63 лет), у мужчин - на 2 года (с 60 до 62 лет).

При рассмотрении результатов факторного анализа у пациентов из IIIа группы здоровья по результатам 1-го этапа диспансеризации в 2015 и 2018 гг. прослеживаются выраженные гендерные различия во вкладе разных модифицируемых ФР ССЗ и их ассоциаций в долю общей дисперсии.
Число выделенных факторов у мужчин с IIIа группой здоровья в 2015 и 2018 г. равно 4, и они соответственно охватывали 59,3\% и 64,1\% общей дисперсии исходных признаков. У женщин с IIIa группой здоровья в 2015 г. число выделенных факторов равно 5, и они охватывали 72,1\% общей дисперсии исходных признаков, а в 2018 г. число факторов снизилось до 4, а их вклад в общую дисперсию - до 60,2\%. Для интерпретации выявленных изменений полезно их сопоставление с частотой модифицируемых ФР ССЗ в динамике за 2015-2018 гг.

У мужчин из IIIа группы здоровья с 2015 по 2018 г. произошли изменения в структуре фактора 1: комбинация АГ и ИМТ > 25 кг/м² сменилась на НП и НФА (ранее входили В фактор 2) (табл. 3, 4). Распространенность АГ у мужчин, прошедших диспансеризацию в 2018 г., возросла; вместе с тем в IIIa группе здоровья произошло ее снижение с 25,1\%

Факторные нагрузки модифицируемых факторов риска ААя мужчин из IIIа группы зАоровья по резумьтатам 1-го этапа Аиспансеризации в 2015 году

Factor weights of modifiable risk factors for male patients in health category IIIa following the results of stage 1 health assessment in 2015

\begin{tabular}{|l|l|l|l|l|}
\hline \multicolumn{1}{|c|}{ Факторы риска / Risk factors } & $\begin{array}{c}\text { Фактор 1 / } \\
\text { Factor 1 }\end{array}$ & $\begin{array}{c}\text { Фактор 2 / } \\
\text { Factor 2 }\end{array}$ & $\begin{array}{c}\text { Фактор 3 / } \\
\text { Factor 3 }\end{array}$ & $\begin{array}{c}\text { Фактор 4 / } \\
\text { Factor 4 }\end{array}$ \\
\hline Курение / Smoking & 0,009 & 0,071 & 0,068 & $-0,778^{* *}$ \\
\hline Риск пагубного потребления алкоголя / Alcohol abuse & $-0,087$ & $-0,144$ & $0,565^{*}$ & $-0,220$ \\
\hline Артериальная гипертензия / Arterial hypertension & $0,785^{* *}$ & $-0,088$ & $-0,158$ & $-0,1168$ \\
\hline Гиперхолестеринемия / Нуреrcholesterolemia & 0,0194 & 0,042 & $0,801^{* *}$ & 0,036 \\
\hline Нерациональное питание / Improper diet & 0,121 & $0,840^{* *}$ & $-0,034$ & $-0,152$ \\
\hline Гипергликемия / Нуреrglycemia & 0,155 & 0,043 & 0,062 & $0,604^{*}$ \\
\hline Низкая физическая активность / Physical inactivity & $-0,230$ & $0,747^{* *}$ & 0,044564 & 0,169 \\
\hline Избыточная масса тела и ожирение / Overweight and obesity & $0,697^{*}$ & 0,078 & 0,225612 & 0,313 \\
\hline Общая дисперсия / Тоtal variance & 1,202 & 1,308 & 1,050 & 1,185 \\
\hline Доля общей дисперсии / Total variance share & 0,150 & 0,163 & 0,131 & 0,148 \\
\hline
\end{tabular}

Примечание. ЗАесь и в таблицах 4, 7, 8: (*) — факторные нагрузки более 0,5 были взяты как значимые А^я интерпретации; (**) — факторные нагрузки более 0,7 были взяты как более строгие Аля интерпретации.

Note. Here and tables 4, 7, 8: $\left(^{*}\right)$ — factor weights $>0.5$ were considered significant for interpretation purposes; $(* *)$ — factor weights $>0.7$ were considered more strict for inetrpretation.

Таблица 4 / Table 4 б

Факторные нагрузки модифицируемых факторов риска Аля мужчин из IIIа группы зАоровья по резуцьтатам 1-го этапа Аиспансеризации в 2018 году

Factor weights of modifiable risk factors for male patients in health category IIIa following the results of stage 1 health assessment in 2018

\begin{tabular}{|l|l|l|l|l|}
\hline \multicolumn{1}{|c|}{ Факторы риска / Risk factors } & $\begin{array}{c}\text { Фактор 1 / } \\
\text { Factor 1 }\end{array}$ & $\begin{array}{c}\text { Фактор 2 / } \\
\text { Factor 2 }\end{array}$ & $\begin{array}{c}\text { Фактор 3 / } \\
\text { Factor 3 }\end{array}$ & $\begin{array}{c}\text { Фактор 4 / } \\
\text { Factor 4 }\end{array}$ \\
\hline Артериальная гипертензия / Arterial hypertension & $-0,137$ & $-0,376$ & $0,603^{*}$ & $-0,538^{*}$ \\
\hline Гиперхолестеринемия / Hypercholesterolemia & $-0,300$ & $-0,519^{*}$ & 0,321 & 0,188 \\
\hline Гипергликемия / Нyperglycemia & $-0,450$ & 0,180 & 0,284 & 0,492 \\
\hline Нерациональное питание / Improper diet & $-0,617^{*}$ & 0,177 & $-0,567^{*}$ & $-0,056$ \\
\hline Низкая физическая активность / Physical inactivity & $-0,743 *$ * & 0,064 & $-0,156$ & $-0,444$ \\
\hline Избыточная масса тела и ожирение / Overweight and obesity & $-0,473$ & $-0,180$ & 0,235 & 0,417 \\
\hline Курение / Sтокіng & 0,135 & $-0,642^{*}$ & $-0,456$ & 0,233 \\
\hline Риск пагубного потребления алкоголя / Alcohol abuse & $-0,048$ & $-0,676^{*}$ & $-0,299$ & $-0,103$ \\
\hline Общая дисперсия / Тотаl variance & 1,491 & 1,382 & 1,247 & 1,008 \\
\hline Доля общей дисперсии / Total variance share & 0,186 & 0,172 & 0,155 & 0,126 \\
\hline
\end{tabular}


до 19,3\% ( $=$ =,0001) (табл. 5, 6). Обращает на себя внимание, что частота ИзМТ и ожирения у мужчин в 2018 г. возросла с $16,46 \%$ до $17,64 \%$, в IIIa группе здоровья - с $22,80 \%$ до $24,43 \%(p=0,247)$ (см. табл. 5, 6).
Структура фактора 2 у мужчин также изменилась: комбинация НП и НФА (перешла в фактор 1) сменилась на сочетание ГХС, курения и РППА (см. табл. 3, 4). Распространенность НП и НФАумужчин в 2018 г. уменьшилась,

Гендерные особенности модифицируемых факторов риска по Аанным 1-го этапа диспансеризации в 2015 году, n (\%)

Gender aspects of modifiable risk factors following the results of stage 1 health assessment in 2015, n (\%)

\begin{tabular}{|c|c|c|c|c|}
\hline \multirow[t]{2}{*}{ Факторы риска / Risk factors } & \multicolumn{2}{|c|}{$\begin{array}{l}\text { Всего за } 2015 \text { r. / Total for } 2015 \\
\qquad(n=2461)\end{array}$} & \multicolumn{2}{|c|}{$\begin{array}{c}\text { IIIa группа здоровья / } \\
\text { Health category IIIa }(n=737)\end{array}$} \\
\hline & муж / males & жен / females & муж / males & жен / females \\
\hline \multirow{2}{*}{$\begin{array}{l}\text { Артериальная гипертензия / Arterial } \\
\text { hypertension }\end{array}$} & \multicolumn{2}{|l|}{$643(26,13)$} & \multicolumn{2}{|l|}{$562(76,25)$} \\
\hline & $218(8,86)$ & $425(17,27)$ & $185(25,10)$ & $377(51,15)$ \\
\hline \multirow{2}{*}{$\begin{array}{l}\text { Гиперхолестеринемия / } \\
\text { Hypercholesterolemia }\end{array}$} & \multicolumn{2}{|l|}{$793(32,22)$} & \multicolumn{2}{|l|}{$413(56,03)$} \\
\hline & $252(10,24)$ & $541(21,98)$ & $141(19,13)$ & $272(36,90)$ \\
\hline \multirow[t]{2}{*}{ Гипергликемия / Hyperglycemia } & \multicolumn{2}{|l|}{$130(5,28)$} & \multicolumn{2}{|l|}{$110(14,93)$} \\
\hline & $41(1,66)$ & $89(3,62)$ & $33(4,48)$ & $77(10,45)$ \\
\hline \multirow[t]{2}{*}{ Нерациональное питание / Improper diet } & \multicolumn{2}{|l|}{$1837(74,64)$} & \multicolumn{2}{|l|}{$595(80,73)$} \\
\hline & $640(26,00)$ & $1197(48,64)$ & $195(26,46)$ & $400(54,27)$ \\
\hline \multirow{2}{*}{$\begin{array}{l}\text { Низкая физическая активность / Physical } \\
\text { inactivity }\end{array}$} & \multicolumn{2}{|l|}{$1321(53,68)$} & \multicolumn{2}{|l|}{$538(72,99)$} \\
\hline & $410(16,66)$ & $911(37,02)$ & $166(22,52)$ & $372(50,47)$ \\
\hline \multirow{2}{*}{$\begin{array}{l}\text { Избыточная масса тела и ожирение / } \\
\text { Overweight and obesity }\end{array}$} & \multicolumn{2}{|l|}{$1096(44,54)$} & \multicolumn{2}{|l|}{$554(75,17)$} \\
\hline & $405(16,46)$ & $691(28,08)$ & $168(22,80)$ & $386(25,37)$ \\
\hline \multirow[t]{2}{*}{ Курение / Smoking } & \multicolumn{2}{|l|}{$248(10,08)$} & \multicolumn{2}{|l|}{$49(6,65)$} \\
\hline & $213(8,66)$ & $35(1,42)$ & $35(4,75)$ & $14(1,90)$ \\
\hline \multirow{2}{*}{$\begin{array}{l}\text { Pиск пагубного потребления алкоголя / } \\
\text { Alcohol abuse }\end{array}$} & \multicolumn{2}{|l|}{$16(0,65)$} & \multicolumn{2}{|l|}{$8(1,09)$} \\
\hline & $6(0,24)$ & $10(0,41)$ & $3(0,41)$ & $5(0,68)$ \\
\hline
\end{tabular}

Таблица 6 / Table 6

Гендерные особенности модифицируемых факторов риска

по данным 1-го этапа диспансеризации в 2018 году, n (\%)

Gender aspects of modifiable risk factors following the results of stage 1 health assessment in 2018, n (\%)

\begin{tabular}{|c|c|c|c|c|}
\hline \multirow[t]{2}{*}{ Факторы риска / Risk factors } & \multicolumn{2}{|c|}{$\begin{array}{l}\text { Всего за } 2018 \text { г. / Total for } 2018 \\
\qquad(n=2772)\end{array}$} & \multicolumn{2}{|c|}{$\begin{array}{c}\text { IIIa группа здоровья / } \\
\text { Health category IIIa }(n=1150)\end{array}$} \\
\hline & муж / males & жен / females & муж / males & жен / females \\
\hline \multirow{2}{*}{$\begin{array}{l}\text { Артериальная гипертензия / Arterial } \\
\text { hypertension }\end{array}$} & \multicolumn{2}{|l|}{$688(24,82)^{*}$} & \multicolumn{2}{|l|}{$611(53,13)$} \\
\hline & $261(9,42)$ & $427(15,40)$ & $222(19,3)^{* *}$ & $389(33,83)^{* *}$ \\
\hline \multirow{2}{*}{$\begin{array}{l}\text { Гиперхолестеринемия / } \\
\text { Hypercholesterolemia }\end{array}$} & \multicolumn{2}{|l|}{$883(31,85)^{*}$} & \multicolumn{2}{|l|}{$552(48,00)$} \\
\hline & $263(9,49)$ & $620(22,36)$ & $173(15,04)^{* *}$ & $379(32,96)^{* *}$ \\
\hline \multirow[t]{2}{*}{ Гипергликемия / Hyperglycemia } & \multicolumn{2}{|l|}{$216(7,79)^{* *}$} & \multicolumn{2}{|l|}{$210(18,26)$} \\
\hline & $84(3,03)$ & $132(4,76)$ & $83\left(7,22^{*}\right)$ & $127(11,04)^{*}$ \\
\hline \multirow[t]{2}{*}{ Нерациональное питание / Improper diet } & \multicolumn{2}{|l|}{$1402(50,58)^{* *}$} & \multicolumn{2}{|l|}{$674(58,61)$} \\
\hline & $484(17,46)$ & $918(33,12)$ & $229(19,91)^{* *}$ & $445(38,70)^{* *}$ \\
\hline \multirow{2}{*}{$\begin{array}{l}\text { Низкая физическая активность / Physical } \\
\text { inactivity }\end{array}$} & \multicolumn{2}{|l|}{$851(30,70)^{* *}$} & \multicolumn{2}{|l|}{$551(47,91)$} \\
\hline & $275(9,92)$ & $576(20,78)$ & $182(15,82)^{* *}$ & $369(32,09)^{* *}$ \\
\hline \multirow{2}{*}{$\begin{array}{l}\text { Избыточная масса тела и ожирение / } \\
\text { Overweight and obesity }\end{array}$} & \multicolumn{2}{|l|}{$1317(47,51)^{* *}$} & \multicolumn{2}{|l|}{$813(70,69)$} \\
\hline & $489(17,64)$ & $828(29,87)$ & $281(24,43)^{*}$ & $532(46,26)^{* *}$ \\
\hline \multirow[t]{2}{*}{ Курение / Smoking } & \multicolumn{2}{|l|}{$135(4,87)^{\star *}$} & \multicolumn{2}{|l|}{$48(4,17)$} \\
\hline & $111(4,00)$ & $24(0,87)$ & $37(3,22)^{* *}$ & $11(0,96)^{*}$ \\
\hline \multirow{2}{*}{$\begin{array}{l}\text { Риск пагубного потребления алкоголя / } \\
\text { Alcohol abuse }\end{array}$} & \multicolumn{2}{|l|}{$9(0,32)^{*}$} & \multicolumn{2}{|l|}{$6(0,52)$} \\
\hline & $5(0,18)$ & $4(0,14)$ & $4(0,35)^{*}$ & $2(0,17)^{*}$ \\
\hline
\end{tabular}

* При $\mathrm{p} \geq 0,05$ принималась нулевая гипотеза об отсутствии разАичий по частоте признака межАу 2015 и 2018 гг.

** При р < 0,05 принималась альтернативная гипотеза о наличии разАичий по частоте признака межАу 2015 и 2018 гг.

* $\mathrm{p} \geq 0.05$ meant a null hypothesis of the lack of differences in the rate of parameter between 2015 and 2018.

** $\mathrm{p}<0.05$ meant an alternative hypothesis of the differences in the rate of parameter between 2015 and 2018. 
в частности в IIIa группе здоровья - с 26,46\% до 19,91\% ( $p=0,0001)$ и с $22,52 \%$ до $15,82 \%(p=0,0001)$ соответственно (см. табл. 5, 6).

В структуре фактора 3 РППА и ГХС (перешли в фактор 2) заменились на АГ и НП, что подчеркивает важность этой ассоциации, несмотря на снижение их частоты у мужчин, прошедших диспансеризацию, и изолированно в IIIa группе здоровья. Распространенность ГХС у мужчин, прошедших диспансеризацию в 2018 г, уменьшилась, в том числе в IIIa группе здоровья (с 19,1\% до 15,04\%; p = 0,014).

Переход комбинации ГХС и РППА из фактора 3 в фактор 2, а также курения из фактора 4 в фактор 2 свидетельствует о растущем вкладе их ассоциации в формирование СС3, несмотря на снижение частоты регистрации данных модифицируемых ФР как изолированно в IIIа группе здоровья, так и в целом у мужчин, прошедших диспансеризацию в 2018 г.

С 2015 по 2018 г. в структуре фактора 4 произошла замена курения (переход в фактор 2) и гипергликемии (снижение факторной нагрузки до 0,492) на АГ (см. табл. 3, 4), что, несмотря на уменьшение ее распространенности с 2015 по 2018 г., подчеркивает важность АГ в качестве изолированного модифицируемого ФР ССЗ.

Частота гипергликемии у мужчин, прошедших диспансеризацию в 2018 г., увеличилась с 1,67\% до 3,03\%, наблюдался ее рост и в IIIa группе здоровья - с 4,48\% до 7,22\% ( $=0,058)$ (см. табл. 5, 6). Некоторое снижение факторной нагрузки гипергликемии (см. табл. 3, 4), невзирая на возрастание ее частоты как в целом, так и в IIIa группе здоровья, может свидетельствовать о росте ее распространенности преимущественно за счет повышения качества выявления.

Присутствие таких модифицируемых ФР, как курение и РППА, в составе 2-го фактора у мужчин в 2018 г. (см. табл. 4) говорит о достаточно сильном их воздействии на течение ССЗ.

У женщин из IIIa группы здоровья за период с 2015 по 2018 г. в структуре фактора 1 комбинация НП, НФА и ИМТ > 25 кг/м² (переместился в фактор 3) сменилась на НП и НФА (табл. 7, 8).

Факторные нагрузки модифицируемых факторов риска Амя женщин из IIIа группы зАоровья по резумьтатам 1-го этапа диспансеризации в 2015 году

Factor weights of modifiable risk factors for female patients in health category IIIa following the results of stage 1 health assessment in 2015

\begin{tabular}{|c|c|c|c|c|c|}
\hline Факторы риска / Risk factors & $\begin{array}{l}\text { Фактор } 1 \text { / } \\
\text { Factor } 1\end{array}$ & $\begin{array}{c}\text { Фактор } 2 \text { / } \\
\text { Factor } 2\end{array}$ & $\begin{array}{l}\text { Фактор } 3 \text { / } \\
\text { Factor } 3\end{array}$ & $\begin{array}{l}\text { Фактор } 4 \text { / } \\
\text { Factor } 4\end{array}$ & $\begin{array}{l}\text { Фактор } 5 / \\
\text { Factor } 5\end{array}$ \\
\hline Артериальная гипертензия / Arterial hypertension & $-0,164$ & $-0,403$ & 0,494 & $-0,096$ & $0,602^{*}$ \\
\hline Гиперхолестеринемия / Hypercholesterolemia & $-0,250$ & 0,062 & 0,037 & $-0,827^{* *}$ & 0,013 \\
\hline Гипергликемия / Hyperglycemia & $-0,060$ & 0,271 & $0,604^{*}$ & 0,012 & $-0,642^{*}$ \\
\hline Нерациональное питание / Improper diet & $-0,647^{*}$ & 0,167 & $-0,517^{*}$ & $-0,012$ & 0,001 \\
\hline Низкая физическая активность / Physical inactivity & $-0,771^{* *}$ & 0,107 & $-0,036$ & 0,219 & $-0,020$ \\
\hline Курение / Smoking & 0,144 & $-0,684^{*}$ & $-0,291$ & 0,223 & $-0,285$ \\
\hline Риск пагубного потребления алкоголя / Alcohol abuse & $-0,268$ & $-0,594^{*}$ & $-0,007$ & $-0,355$ & $-0,383$ \\
\hline $\begin{array}{l}\text { Избыточная масса тела и ожирение / Overweight } \\
\text { and obesity }\end{array}$ & $-0,507^{*}$ & $-0,189$ & 0,377 & 0,372 & 0,024 \\
\hline Общая дисперсия / Total variance & 1,458 & 1,137 & 1,108 & 1,057 & 1,005 \\
\hline Доля общей дисперсии / Total variance share & 0,182 & 0,142 & 0,138 & 0,132 & 0,125 \\
\hline
\end{tabular}

Таблица 8 / Table 8 б

Факторные нагрузки модифицируемых факторов риска Аһя женщин из IIIа группы зАоровья по резумьтатам 1-го этапа диспансеризации в 2018 году

Factor weights of modifiable risk factors for female patients in health category IIIa following the results of stage 1 health assessment in 2018

\begin{tabular}{|l|l|l|l|l|}
\hline \multicolumn{1}{|c|}{ Факторы риска / Risk factors } & $\begin{array}{c}\text { Фактор 1 / } \\
\text { Factor 1 }\end{array}$ & $\begin{array}{c}\text { Фактор 2 / } \\
\text { Factor 2 }\end{array}$ & $\begin{array}{c}\text { Фактор 3 / } \\
\text { Factor 3 }\end{array}$ & $\begin{array}{c}\text { Фактор 4 / } \\
\text { Factor 4 }\end{array}$ \\
\hline Риск пагубного потребления алкоголя / Alcohol abuse & $-0,006$ & $-0,022$ & $-0,014$ & $0,960^{* *}$ \\
\hline Курение/ Smoking & $-0,026$ & 0,217 & 0,269 & 0,035 \\
\hline Избыточная масса тела и ожирение / Overweight and obesity & 0,018 & 0,248 & $0,663^{*}$ & 0,194 \\
\hline Артериальная гипертензия / Arterial hypertension & $-0,175$ & $0,769 * *$ & $-0,005$ & $-0,085$ \\
\hline Гиперхолестеринемия / Hypercholesterolemia & 0,215 & $0,645^{*}$ & $-0,054$ & 0,064 \\
\hline Гипергликемия / Нуреrglycemia & 0,056 & $-0,187$ & $0,772^{* *}$ & $-0,147$ \\
\hline Нерациональное питание / Improper diet & $0,850^{* *}$ & $-0,194$ & 0,013 & 0,098 \\
\hline Низкая физическая активность / Physical inactivity & $0,811^{* *}$ & 0,171 & 0,050 & $-0,128$ \\
\hline Общая дисперсия / Тоtal variance & 1,462 & 1,221 & 1,116 & 1,021 \\
\hline Доля общей дисперсии / Total variance share & 0,182 & 0,125 & 0,139 & 0,127 \\
\hline
\end{tabular}


Частота НП и НФА у женщин в 2018 г. уменьшилась как в целом, так и в IIIa группе здоровья - с 54,27\% до $38,70 \%$ и с $50,47 \%$ до 32,09\% (в обоих случаях $\mathrm{p}=0,0001$ ) (см. табл. 5, 6). Обращает на себя внимание, что доля пациенток с ИзМТ и ожирением среди женщин в целом возросла, а в IIIa группе здоровья снизилась с 52,37\% до 46,26\% ( $p=0,015)$. За период с 2015 по 2018 г. у женщин в структуре фактора 2 также произошли изменения: комбинация курения (уменьшение факторной нагрузки) и РППА (переместилось в фактор 4) сменилась на АГ и ГХС (см. табл. 7, 8).

Гораздо более низкая распространенность курения и РППА у мужчин и женщин, прошедших диспансеризацию в 2015 и 2018 г. (см. табл. 5, 6), по сравнению с таковой по результатам ЭССЕ-РФ [11] свидетельствует о необходимости повышения качества их выявления. Присутствие модифицируемого ФР (РППА) в составе 4-го фактора у женщин в 2018 г. (см. табл. 8) предположительно указывает на достаточно сильное его влияние на течение ССЗ у лиц, злоупотребляющих алкоголем.

За период с 2015 по 2018 г. в структуре фактора 3 комбинация гипергликемии и НП сменилась на сочетание ИзМТ и ожирения и гипергликемии (см. табл. 7, 8), что подчеркивает важность указанной ассоциации, несмотря на некоторое снижение их частоты у женщин IIIa группы здоровья. Распространенность гипергликемии в 2018 г. возросла у женщин в целом и в IIIa группе здоровья - с 10,45\% до $11,04 \%(p=0,36)$ (см. табл. 5, 6).

В структуре фактора 4 у женщин произошла смена ГХС (переместилась в фактор 2) на РППА (см. табл. 7, 8). Частота ГХС у женщин в 2018 г. в целом увеличилась, а в IIIa группе здоровья, напротив, уменьшилась с 39,62\% до $32,96 \%(p=0,023)$.

В 2015 г. в состав фактора 5 у женщин входили АГ (переместилась в фактор 2) и гипергликемия (в факторе 3 ее факторная нагрузка возросла с 0,604 до 0,772), но 2018 г. произошла его ликвидация за счет перераспределения входивших в него модифицируемых ФР ССЗ по факторам 2 и 3 (см. табл. 7, 8).

\section{ОБСУЖДЕНИЕ}

В настоящее время установлено, что вероятность развития СС3 определяется наследственной предрасположенностью и комбинацией модифицируемых ФР [12, 13]. Поэтому при поиске новых подходов к оптимизации профилактики ССЗ актуально выявление структуры сочетаний и скрытых взаимосвязей между модифицируемыми ФР с помощью метода главных компонент. Согласно данным литературы, этот подход широко применяется для разработки мер профилактики СС3, преимущественно за счет поиска и оценки взаимосвязей компонентов метаболического синдрома и отдельных модифицируемых ФР [14]. Если рассматривать дифференцированный подход к профилактике ССЗ с учетом групп здоро-

\section{ЛИТЕPATYPA/REFERENCES}

1. Калинина А.М., Кушунина Д.В., Горный Б.Э. и др. Потенциал профилактики сердечно-сосудистых заболеваний по результа там диспансеризации взрослого населения. Кардиоваскулярная терапия и профилактика. 2019; 18(4): 69-73. [Kalinina A.M., Kushunina D.V., Gornyi B.E. et al. The potential of cardiovascular diseases' prevention according to the results of dispensary examinations of the adult population. Cardiovascular Therapy and Prevention. 2019; 18(4): 69-76. (in Russian)]. DOI: 10.15829/1728-8800-2019-4-69-76

2. Драпкина О.М., Самородская И.В., Старинская М.А. и др. Сравнение российских регионов по уровню стандартизован вья, то в IIIа группе здоровья фокус при разработке мер профилактики СС3 смещается на предотвращение дальнейшего прогрессирования заболеваний сердечно-сосудистого континуума, в том числе острых сосудистых катастроф и вызванных ими летальных исходов и инвалидизации.

Согласно полученным нами данным, общая дисперсия, обусловленная факторами, объединяющими в себе модифицируемые ФР, варьирует от 59,3\% до 72,1\%. При сопоставлении с данными литературы можно найти аналогичные цифры (около 50\%), что позволяет сделать заключение об их значимости для развития неблагоприятных сердечно-сосудистых событий у пациентов из IIIa группы здоровья [15].

При анализе данных 1-го этапа диспансеризации с помощью метода главных компонент у пациентов обоих полов c IIIa группой здоровья прежде всего обращают на себя внимание изменения в структуре 1-го фактора. Так с 2015 по 2018 г. как у мужчин (АГ и ИМТ > 25 кг/м²), так и у жен-

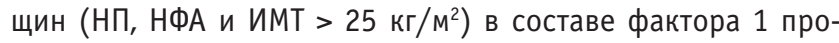
изошла замена на комбинацию НП и НФА. При этом НП и НФА потенцируют повышение массы тела и развитие ожирения, дислипидемии, АГ и нарушений углеводного обмена. Перечисленные состояния тесно связаны с формированием и прогрессированием ССЗ и обусловленной ими преждевременной смертностью $[16,17]$.

\section{ЗАКЛЮЧЕНИЕ}

Возрастные и гендерные особенности результатов 1-го этапа диспансеризации в 2015 и 2018 г. свидетельствуют о необходимости увеличения охвата населения диспансеризацией с акцентом на повышение привлечения мужчин.

При проведении 1-го этапа диспансеризации следует уделить особое внимание эффективному и своевременному выявлению IIIa группы здоровья в целевой группе населения: у женщин в возрасте 56-71 год, а у мужчин - в возрасте 53-69 лет.

Результаты факторного анализа модифицируемых факторов риска (ФР) болезней системы кровообращения у пациентов с IIIа группой здоровья позволяют проводить мероприятия по коррекции ФР с учетом сочетаний, оказывающих наиболее значимое влияние на наступление неблагоприятных сердечно-сосудистых событий. У мужчин фактор 1 нерациональное питание (НП) и низкая физическая активность (НФА); фактор 2 - гиперхолестеринемия (ГХС), курение табака и риск пагубного потребления алкоголя (РППА); фактор 3 - АГ и НП; фактор 4 - АГ; у женщин фактор 1 НП и НФА; фактор 2 - АГ и ГХС; фактор 3 - избыточная масса тела и ожирение и гипергликемия; фактор 4 - РППА.

С учетом крайне низкой частоты выявления курения табака и злоупотребления алкоголем у пациентов C IIIa группой здоровья необходимо акцентировать внимание на повышении эффективности мероприятий по их обнаружению и коррекции с позиции «политики нулевой терпимости».

ных коэффициентов смертности от всех причин и болезней системы кровообращения в 2006-2016 г2. Профилактическая медицина. 2018; 21(4): 4-12. [Drapkina 0.M., Samorodskaya I.V., Starinskaya M.A. et al. Comparison of Russian regions by standardized mortality rates from all causes and circulatory system diseases in 2006-2016. Russian Journal of Preventive Medicine. 2018; 21(4): 4-12. (in Russian)]. DOI: 10.17116/profmed20182144

3. Скворцова В.И., Шетова И.М., Какорина Е.П. и др. Снижение смертности от острых нарушений мозгового кровообращения в результате реализации комплекса мероприятий по совершенствованию медицинской помощи пациентам с сосудистыми заболеваниями в Российской Федерации. Профилактическая 
медицина. 2018; 21(1): 4-10. [Skvortsova V.I., Shetova I.M., Kakorina E.P. et al. Reduction in stroke death rates through a package of measures to improve medical care for patients with vascular diseases in the Russian Federation. Russian Journal of Preventive Medicine. 2018; 21(1): 4-10. (in Russian)]. DOI: 10.17116/profmed20182114-10

4. Калинина А.М., Кушунина Д.В., Горный Б.Э. Повышение качества диспансеризации взрослого населения как важный инструмент профилактики сердечно-сосудистых заболеваний в первичном звене здравоохранения. Профилактическая медицина. 2018; 21(5): 22-7. [Kalinina A.M., Kushunina D.V., Gornyi B.E. The tasks of improving the quality of medical examination of the adult population as an important tool to prevent cardiovascular diseases in primary health care. Russian Journal of Preventive Medicine. 2018; 21(5): 22-7. (in Russian)]. DOI: 10.17116/profmed20182105122

5. Coke L.A., Himmelfarb C.D. Guideline on the primary prevention of cardiovascular disease: let's get it into practice! J. Cardiovasc. Nurs. 2019; 34(4): 285-8. DOI: 10.1161/CIR.0000000000000678

6. Самородская И.В., Ларина В.Н., Бойцов С.А. Вклад четырех групп неинфекционных заболеваний в смертность населения регионов Российской Федерации в 2015 г. Профилактическая медицина. 2018; 21(1): 32-8. [Samorodskaya I.V., Larina V.N., Boitsov S.A. Contribution of four groups of noncommunicable diseases to mortality in the Russian Federation in 2015. Russian Journal of Preventive Medicine. 2018; 21(1): 32-8. (in Russian)]. doi.org/10.17116/profmed201821132-38

7. Piepoli M.F., Hoes A.W. , Agewall S. et al. Европейские рекомендации по профилактике сердечно-сосудистых заболеваний в клинической практике (пересмотр 2016). Российский кардиологический журнал. 2017; 6(146): 7-85. [Piepoli M.F., Hoes A.W., Agewall S. et al. 2016 European guidelines on cardiovascular disease prevention in clinical practice. Russian Journal of Cardiology. 2017; 6(146): 7-85. (in Russian)]. DOI: 10.15829/1560-4071-2017-6-7-85

8. Гржибовский А.М. Анализ номинальных данных (независимые наблюдения). Экология человека. 2008; 6: 58-68. [Grjibowski A.M. Analysis of nominal data (independent observations). Human Ecology. 2008; 6: 58-68. (in Russian)]

9. Hair J.F., Black W.C., Babin B.J. et al. Multivariate data analysis. $7^{\text {th }}$ ed. Edinburgh: Pearson Education Limited; 2014. 816 p.

10. O'Rourke N., Hatcher L. A step-by-step approach to using $S A S^{\circledR}$ for factor analysis and structural equation modeling. 2nd ed. Cary, NC: SAS Institute Inc.; 2013. 408 p.

11. Остроумова О.Д., Извеков А.А., Воеводина Н.Ю. Курение как фактор риска сердечно-сосудистых и цереброваскулярных заболеваний: распространенность, влияние на прогноз, возможные стратегии прекращения курения и их эффективность. Часть 1. Распространенность курения и влияние на прогноз. Рациональная фармакотерапия в кардиологии. 2017; 13(6): 871-9. [Ostroumova O.D., Izvekov A.A., Voevodina N.Yu. Smoking as a risk factor of cardiovascular and cerebrovascular diseases: prevalence, impact on prognosis, possible smoking cessation strategies and their effectiveness. Part 1. Smoking prevalence and impact on prognosis. Rational Pharmacotherapy in Cardiology 2017; 13(6): 871-9. (in Russian)]. DOI: 10.20996/1819-6446-2017-136-871-879

12. Калинина А.М., Горный Б.Э., Кушунина Д.В. и др. Интегральная оценка потенциала медицинской профилактики сердечно-сосудистых заболеваний по результатам диспансеризации и ее прогностическая значимость. Кардиоваскулярная терапия и профилактика. 2020; 19(3): 2547. [Kalinina A.M., Gornyi B.E., Kushunina D.V. et al. Integrated assessment of the potential for cardiovascular prevention according to the screening results and its prognostic significance. Cardiovascular Therapy and Prevention. 2020; 19(3): 2547. (in Russian)]. DOI: 10.15829/1728-88002020-2547

13. Tikkanen E., Gustafsson S., Ingelsson E. Associations of fitness, physical activity, strength, and geneticrisk with cardiovasculardisease: longitudinal analyses in the UK Biobank Study. Circulation. 2018; 137(24): 2583-91. DOI: 10.1161/CIRCULATIONAHA.117.032432

14. Масленникова Г.Я., Оганов Р.Г. Выбор оптимальных подходов к профилактике неинфекционных заболеваний в рамках международного сотрудничества. Кардиоваскулярная терапия и профилактика. 2018; 17(1): 4-9. [Maslennikova G.Ya., Oganov R.G. Selection of optimal approaches to prevention of non-communicable diseases in international partnership circumstances. Cardiovascular Therapy and Prevention. 2018; 17(1): 4-9. (in Russian)]. DOI: 10.15829/1728-8800-2018-1-4-9

15. Tang W., Miller M.B., Rich S.S. et al. Linkage analysis of a composite factor for the multiple metabolic syndrome: the National Heart, Lung, and Blood Institute Family Heart Study. Diabetes. 2003; 52(11): 2840-7. DOI: 10.2337/diabetes.52.11.2840

16. Hillier T.A., Rousseau A., Lange C. et al. Practical way to assess metabolic syndrome using a continuous score obtained from principal components analysis. Diabetologia. 2006; 49(7): 152835. DOI: 10.1007/s00125-006-0266-8

17. Atkins J.L., Whincup P.H., Morris R.W. et al. Dietary patterns and the risk of CVD and all-cause mortality in older British men. Br. J. Nutr. 2016; 116(7): 1246-55. DOI: 10.1017/S0007114516003147 D 